\title{
Contribución de la prensa a la adquisición del vocabulario
}

\section{Contribution of the press to the acquisition of vocabulary}

\author{
Inmaculada Clotilde Santos Díaz \\ Universidad de Málaga (España) \\ santosdiaz@uma.es \\ Ester Trigo Ibáñez \\ Universidad de Cádiz (España) \\ ester.trigo@uca.es \\ Manuel Francisco Romero Oliva \\ Universidad de Cádiz (España) \\ manuelfrancisco.romero@uca.es
}

\begin{abstract}
The press brings the reader closer not only to the news of a community but also to its language and culture. The objective of this study is to know the relationship between press consumption and lexical competence. Specifically, the weekly time spent by 171 graduate students from Malaga reading the press and its relationship with two lexical tests in Spanish - mother tongue - and French or English - foreign language will be analysed. The results show that there is no significant relationship between available vocabulary and press consumption. Instead, the relationship is significant with the ability to identify technicalities when the data refer to the foreign language of the informants. These findings invite us to continue promoting didactic applications that integrate press reading both to improve lexical competence and to integrate the sociopragmatic component of the language.
\end{abstract}

Keywords: language teaching, lexical competence, media, sociolinguistics 


\section{INTRODUCCIÓN}

La utilización de la prensa en la enseñanza y aprendizaje de una lengua extranjera ha sido muy recomendada por la investigación científica principalmente porque resulta un "recurso dinámico y dinamizador que permite poner a los alumnos en contacto con la realidad y comprender mejor las transformaciones del entorno" (Fernández, Fernández-Jodar, 2009, p. 67), lo que, sin duda, aumentará la motivación de los aprendientes (Parratt, 2010; Spychała-Wawrzyniak, Sagermann Bustinza, 2016) al acercarles al uso pragmático del vocabulario y a la realidad socio-cultural del contexto estudiado.

De acuerdo con esto, igual que los textos literarios en la enseñanza de español como lengua extranjera (en adelante, ELE) les aproximan -desde un enfoque intercultural- al patrimonio artístico y social del ámbito hispanoparlante (Trigo, Romero, 2017; Villegas-Paredes, 2020) y conectan con el mundo emocional de los estudiantes (Spychała-Wawrzyniak, 2019), la lectura de textos periodísticos se erige en una ventana hacia la comprensión del contexto donde estos se emiten, pues les permitirá adentrarse en las problemáticas más acuciantes, conocer la evolución política, emitir juicios críticos sobre el discurrir de la economía, la gestión realizada en los momentos de crisis, etc., ya que el discurso periodístico puede ser tanto informativo como argumentativo. Además, recoge un léxico claro, conciso y generalmente correcto, que puede llegar a ser especializado según el tema abordado. Aunque se suele emplear el estilo directo, no está exento del componente retórico, lo que brinda una oportunidad para explotar su potencial cognitivo, pragmático y metafórico en el aula de ELE.

Sin embargo, esto solo podrá suceder si los docentes son conscientes de que llevar la prensa al aula debe perseguir el objetivo de mejorar el comportamiento lingüístico de los discentes mediante la integración de las distintas destrezas comunicativas y sociopragmáticas (Romero, Jiménez, 2015). Por ello, consideramos que las "fases para una gramática pedagógica” propuestas por Romero, Jiménez, Trigo (2020, p. 127) garantizan un aprendizaje compartido y activo en el aula, además de una estrategia para elaborar discursos críticos sobre la realidad.

A este respecto cobra especial importancia el vocabulario emergente. Pongamos como ejemplo la crisis originada por la COVID-19 y su irrupción en la prensa española y, por ende, en el vocabulario activo de los hablantes (Ávila, Santos-Díaz, Trigo, 2020). Una rápida mirada a los titulares más recientes ${ }^{1}$ dejará ver palabras como: desescalada, confinamiento, rebrote, rastreadores, negacionismo, cuarentena, etc. Se trata de un lé-

${ }^{1}$ Se han tomado, como ejemplo, titulares de los periódicos de mayor tirada en España. Así, El País alude a la desescalada: https://elpais.com/ciencia/2020-09-24/las-lecciones-internacionales-de-la-desescalada-senalan-las-flaquezas-de-espana.html; El Mundo, a los rastreadores: https://www.elmundo.es/ ciencia-y-salud/salud/2020/09/27/5f703184fc6c83a45b8b45d5.html; El ABC, al negacionismo: https:// sevilla.abc.es/sociedad/abci-alargada-sombra-negacionismo-historia-interminable-lucha-contra-razon-y-ciencia-202009270153_noticia.html; o La Vanguardia, a los rebrotes: https://www.lavanguardia. com/vida/20200927/483699329778/datos-covid-catalunya.html. 
xico muy actualizado y de alto impacto social pues, en los medios de comunicación se muestra un lenguaje actual y el vocabulario más usado en un momento preciso por los informantes de una determinada comunidad de habla. Quizás en los manuales de ELE los términos rebrote o confinamiento ni siquiera aparezcan en los niveles de usuario básico (A1 y A2) y usuario independiente (B1 y B2) del Marco Común Europeo de Referencia de las Lenguas (Consejo de Europa, 2001). En cambio, dada la situación mundial actual, existe una serie de vocablos ligados a la pandemia que forman parte de las conversaciones cotidianas de los informantes -mascarilla, gel hidroalcohólico, test serológico, $P C R$, coronavirus, entre otras- que hace unos meses serían muy poco usados.

Esta idea nos lleva a recurrir a los estudios de disponibilidad léxica, iniciados en la década de los 60 de la pasada centuria en Francia por Gougenheim, Michéa, Rivenc, Sauvageot (1956) bajo el convencimiento de que las lenguas cuentan con un amplio número de lexías de alto contenido semántico cuya aparición está directamente relacionada con el tema del discurso. Así, estos lingüistas comenzaron a aplicar pruebas de léxico asociativo en las que los informantes debían escribir, en un tiempo determinado, todas las palabas que afloraran a su mente dado un centro de interés. Siendo el índice de disponibilidad léxica una medida que combina frecuencia y orden de aparición, se considera que las palabras más disponibles serán las que los estudiantes de una lengua aprenderán con mayor facilidad. Centrándonos en la lengua española, gracias a la existencia de un Proyecto Panhispánico de Disponibilidad Léxica, dirigido por Humberto López Morales, estos estudios han cobrado gran importancia y han ido avanzando metodológicamente (López-González, 2014). De hecho, existe toda una corriente centrada en la enseñanza de ELE (Samper-Hernández, 2002; Šifrar, 2014) y en la evaluación de la competencia léxica de los futuros docentes (Herranz, 2020; Santos-Díaz, 2020).

Consideramos que, para aprender correctamente una lengua, la exposición a esta resulta un factor clave, ya sea de forma activa o pasiva, oral o escrita. En lo que respecta a las distintas prácticas relacionadas con la lectura, se ha constatado, en estudios previos, que tienen una incidencia positiva en la comprensión lectora y el aumento del vocabulario tanto en lengua materna (Castillo-Fadić, Sologuren, 2020; Juárez, 2019) como en lengua extranjera (Brown, Waring, Donkaewbua, 2008; Jiménez-López, Santos-Díaz, Trigo, 2019). Además, desde el ámbito de la disponibilidad léxica, se ha comprobado que los estudiantes más lectores pueden reconocer más vocablos especializados (Cepeda et al., 2017; Santos-Díaz, 2017). Sin embargo, consideramos que existe un vacío referido a los estudios que relacionen la lectura de prensa con el léxico disponible de los informantes.

Por ello, este estudio parte de la hipótesis de que la exposición a diferentes situaciones comunicativas incide en la competencia lingüística y, por ende, en el vocabulario de los hablantes. Su principal objetivo es conocer la relación entre el consumo lector de prensa, una actividad cotidiana y social entre los hablantes y aprendices de lenguas, con el léxico, según se trate de la lengua materna o extranjera del informante. 


\section{METODOLOGÍA}

\subsection{PARTICIPANTES}

La población está formada por los estudiantes del Máster en Profesorado de Educación Secundaria Obligatoria, Bachillerato, Formación Profesional y enseñanza de idiomas de la Universidad de Málaga durante el curso 2010-2011. Concretamente, realizaron las pruebas 171 estudiantes de un total de 386 matriculados, siguiendo los criterios de representatividad y validez de Dörnyei \& Csizér (2012). Todos los participantes acreditaron un nivel mínimo de B1 en lengua extranjera, concretamente 150 lo hicieron en inglés y 21 , en francés. La edad media de los informantes es de 28,73. Han participado más mujeres $(\mathrm{n}=117)$ que hombres $(\mathrm{n}=54)$. Para la mayoría de los informantes el español es su lengua materna $(n=160)$, pero también hay informantes bilingües $(n=4)$ y otros cuya lengua materna es otra lengua no presente en las pruebas $(n=7)$ y que tienen el español como lengua extranjera ${ }^{2}$.

\subsection{INSTRUMENTO}

Atendiendo a los objetivos, se realizaron tres cuestionarios complementarios:

a) Cuestionario sociodemográfico y con preguntas relacionadas con la exposición a una lengua. En esta parte, se recogieron los datos que ayudan a definir la muestra: el sexo, edad y lengua materna y, además, se formularon las preguntas relativas al consumo de prensa en forma de escala de Likert. Los estudiantes debían marcar con una cruz la opción del número de horas semanales que dedican a leer prensa distinguiendo entre el español o la lengua extranjera en la que realizaron posteriormente las pruebas lingüísticas (francés o inglés): 1) ninguna hora; 2) menos de dos horas; 3 ) entre dos y cinco horas y 4) más de cinco horas.

b) Prueba de disponibilidad léxica. Se siguieron las pautas metodológicas más comunes (López-González, 2014) en la que los informantes deben escribir en forma de columna todas las palabras que se le vengan a la mente relacionadas con un tema o centro de interés. El tiempo para cada centro de interés es de dos minutos y se seleccionaron nueve, iguales en español y en lengua extranjera (francés o inglés), de los cuales ocho pertenecen a los clásicos presentes en los trabajos pioneros (Gougenheim et al., 1956): 1) Partes del cuerpo humano; 2) La ropa; 3) Comidas y bebidas; 4) La escuela (muebles y materiales); 5) La ciudad; 6) Medios de transporte; 7) Juegos y distracciones; 8) Profesiones y oficios; y además,

${ }^{2}$ Se tomará el concepto de "lengua extranjera" en sentido amplio, ya que se desconocen las peculiaridades en el aprendizaje y adquisición de la lengua española en el caso de estos estudiantes, pudiendo haberla aprendido en la mayoría de los casos como segunda lengua. 
9) Ordenadores e internet, que es de creación propia debido a su estrecha relación con la profesión docente.

c) Test de identificación de tecnicismos. En esta prueba los estudiantes tenían que clasificar y relacionar con los centros de interés anteriormente mencionados un total de 18 palabras pertenecientes a un lenguaje más técnico o especializado, tales como conurbación, proctólogo o taxidermista. Igual que en la prueba anterior, en lengua extranjera se ofreció la opción de elegir entre francés o inglés, según la lengua acreditada para el acceso al máster.

\subsection{PROCEDIMIENTO}

Tras recoger los datos presencialmente y en papel, se realizó un plan de codificación para el cuestionario, se lematizaron las palabras de la prueba de léxico disponible siguiendo las directrices de Santos-Díaz (2020) y se corrigió la prueba de léxico especializado teniendo en cuenta la aplicación de un factor de corrección probabilístico en el caso de respuestas incorrectas. Asimismo, se contabilizaron todas las palabras escritas por los informantes en los diferentes centros de interés según la lengua de la prueba.

Para el análisis de los datos se utilizó el paquete estadístico IBM SPSS Statistics (v. 22) teniendo en cuenta las siguientes variables: número de palabras consignadas en la prueba de disponibilidad léxica, resultado de la prueba de identificación de tecnicismos, tiempo semanal dedicado a lectura de la prensa y lengua materna de los informantes. Se ha segmentado la muestra según el tipo de lengua de los estudiantes: español (en adelante ELM), español como lengua extranjera (ELE) y bilingües (BL) -español y otra lengua-. Sin embargo, se han desestimado los datos de los estudiantes BL para eliminar posibles interferencias en los resultados debido a las casuísticas particulares de los informantes, ya que no compartían los mismos idiomas (catalán, gallego, inglés y árabe). Las pruebas estadísticas aplicadas serán de tipo descriptivo (distribución de frecuencias, medias según subgrupo) e inferencial (comparación de medias entre dos grupos).

\section{ANÁLISIS}

\subsection{EL CONSUMO DE PRENSA}

Para obtener una visión general del consumo de prensa entre el futuro profesorado, la Figura 1 recoge la frecuencia porcentual obtenida en esa pregunta según la lengua materna de los informantes y el idioma en que lo leen. En español, el porcentaje de estudiantes que no dedica ninguna hora a la semana a leer la prensa es similar 
tanto en los estudiantes de ELM (28,13\%) como en los de ELE $(28,57 \%)$. La pauta más común en los estudiantes ELM es de menos de dos horas $(49,38 \%)$ mientras que en los de ELE la distribución es más homogénea en todas las opciones de la pregunta.

En cambio, cuando se trata de la lectura de prensa en lengua extranjera, el porcentaje es ligeramente superior en el subgrupo de los estudiantes ELM $(86,24 \%)$ que ELE (71,43\%), mostrando en ambos casos el escaso contacto de los estudiantes con los medios de comunicación en una lengua diferente al español. Tan solo un 8,75\% de los informantes ELM afirma que dedica más de cinco horas a la semana a leer en español y un $1,25 \%$ en lengua extranjera. En cuanto a los estudiantes ELE, el porcentaje es mayor en ambos subgrupos $(14,29 \%)$.

¿Cuántas horas a la semana dedicas a leer prensa?

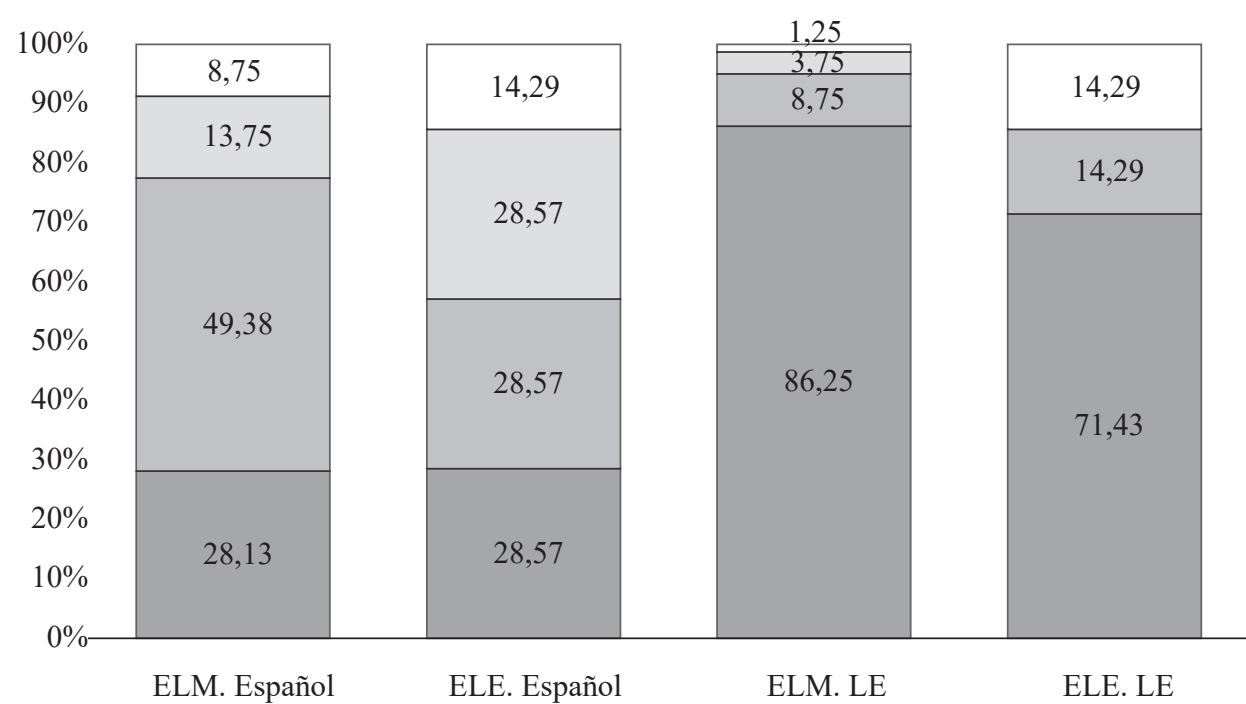

$\square$ Ninguna hora a la semana $\square$ Menos de $2 \quad \square$ Entre 2 y $5 \quad \square$ Más de 5

Fig. 1. Horas semanales dedicadas a leer prensa (Elaboración propia)

A continuación, se ha realizado una comparación de medias teniendo en cuenta como variable dependiente el número total de palabras aportadas en la prueba de léxico disponible según el idioma de la prueba y como variable independiente el número de horas semanales que los estudiantes dedican a leer prensa semanalmente. La Figura 2 muestra que la media de palabras más baja consignada por los informantes suele situarse entre los que no leen ninguna hora a la semana en los dos idiomas. No obstante, se constata que, en español, la línea que se corresponde con los estudiantes ELM es casi plana, mostrando que la lectura de prensa no marca ninguna diferencia en la producción léxica. Sin embargo, cuando se trata de estudiantes ELE, hay un au- 
mento considerable del número de palabras en aquellos que leen más de cinco horas a la semana prensa.

En cuanto a la lengua extranjera, tanto los estudiantes ELM como ELE muestran un comportamiento similar en sus respuestas: los informantes que no leen prensa han escrito un número ligeramente inferior de palabras que aquellos que dedican alguna hora semanal. Sin embargo, no se percibe una progresión clara según el número de horas que dedican, ya que el promedio de palabras es similar en los tres intervalos (menos de dos horas, entre dos y cinco horas y más de cinco horas).

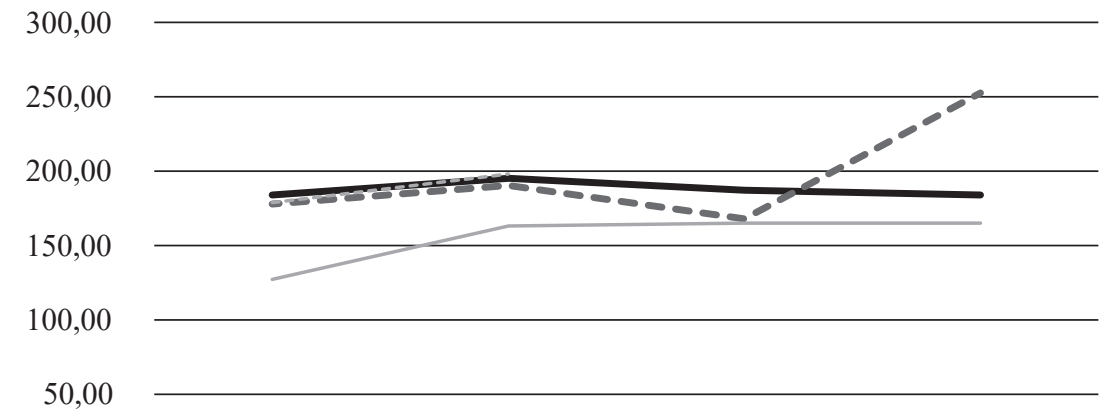

\begin{tabular}{|l|c|c|c|c|}
\cline { 2 - 5 } \multicolumn{1}{c|}{} & $\begin{array}{c}\text { Ninguna hora } \\
\text { a la semana }\end{array}$ & Menos de 2 & Entre 2 y 5 & Más de 5 \\
\hline - ELM. Español & 183,89 & 195,11 & 187,09 & 184,00 \\
\hline - - - ELE. Español & 178,00 & 190,50 & 168,00 & 252,50 \\
\hline - ELM. LE & 127,30 & 163,00 & 164,83 & 164,92 \\
\hline ----- ELE. LE & 178,80 & 198,00 & & 203,00 \\
\hline
\end{tabular}

Fig. 2. El léxico disponible según el consumo de prensa (Elaboración propia)

Para comprobar si la diferencia entre medias se debe al azar o si, por el contrario, existe alguna relación entre ambas variables, se ha realizado una comparación muestral utilizando el estadístico T para muestras independientes. Atendiendo a los resultados anteriores, antes de aplicar la prueba se ha recodificado la variable relativa al consumo de prensa y se han dicotomizado las respuestas, distinguiendo entre "no lee prensa" y "sí lee prensa" - con independencia de las horas que cada informante dedique-. Asimismo, se ha realizado solo con los estudiantes de ELM debido al escaso número de informantes de ELE. En ambas lenguas no se ha podido asumir que las varianzas sean iguales porque el estadístico de la prueba de Levene era $<0,05$. Por tanto, la variable relacionada con la lectura de prensa no tiene una relación significativa a nivel estadístico con el léxico disponible. 


\subsection{RELACIÓN ENTRE EL CONSUMO DE PRENSA Y LA CAPACIDAD DE IDENTIFICACIÓN DEL LÉXICO ESPECIALIZADO}

Siguiendo la dinámica anterior, la Figura 3 recoge el promedio de los resultados obtenidos en la prueba de identificación de tecnicismos en función del número de horas que dedican a leer prensa a la semana. Igual que en el caso anterior, se ha calculado diferenciando el tipo de lengua de los informantes y la lengua de la prueba. En español, los resultados de los estudiantes ELM casi dibujan una línea recta, dejando constancia de la similitud en sus respuestas. Para los estudiantes de ELE sí se observa cierto aumento, teniendo en cuenta que esa mayor exposición a la lengua puede interferir en un mejor conocimiento léxico.

En lengua extranjera, en ambos subgrupos se observa una mayor progresión entre los estudiantes que no dedican ninguna hora con respecto al resto. Los de ELM pasan de una media de 2,68 puntos cuando no dedican a leer ninguna hora a más de 4 puntos en el resto de los casos. En los estudiantes de ELE, esa progresión es aún mayor, ya que oscila entre 2,73 y 9,52 puntos. Cabe mencionar que salvo en el caso específico del estudiante de ELE que dedica más de 5 horas a leer en lengua extranjera, el resto prácticamente no llega a 5 puntos en la prueba. La puntuación máxima de la prueba puede llegar a 10 puntos, siendo 5 la puntuación mínima que, en el ámbito universitario español, se debe alcanzar para aprobar.

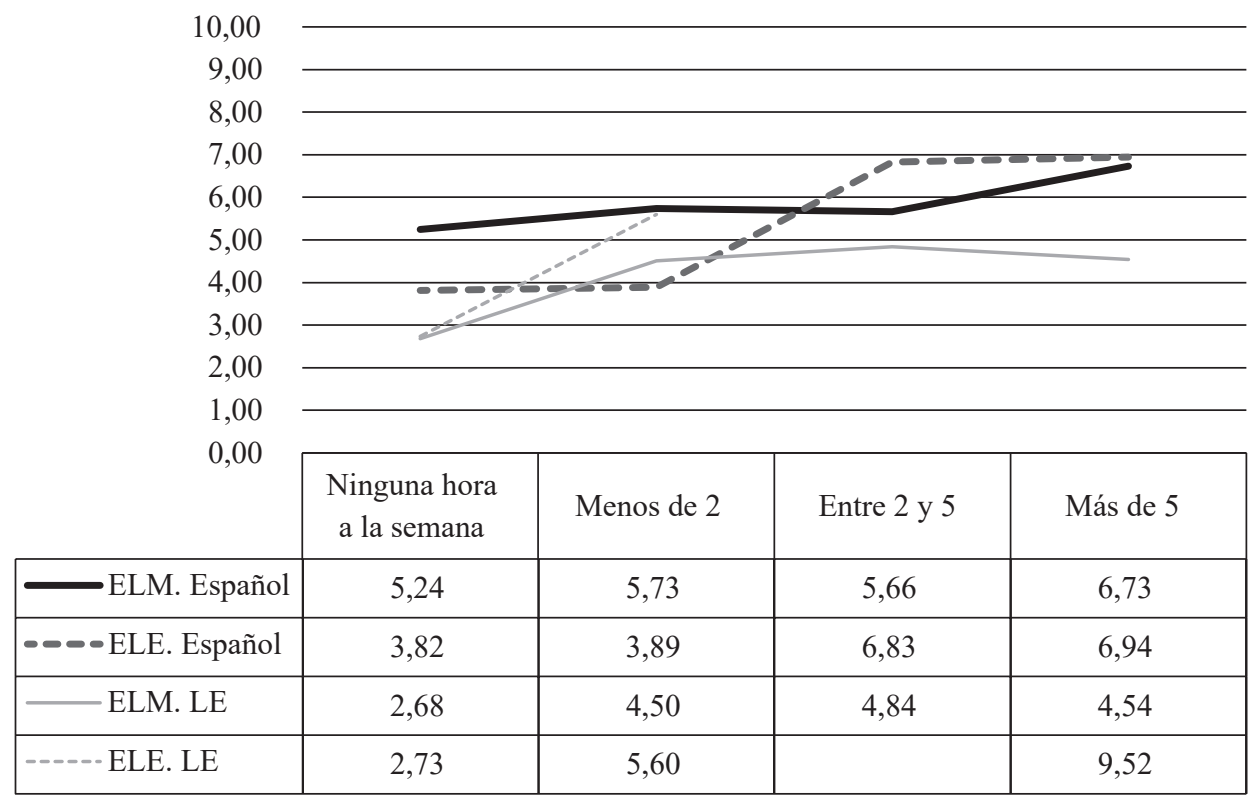

Fig. 3. La capacidad de identificación de tecnicismos según el consumo de prensa (Elaboraciónp propia) 
La Tabla 1 muestra que la prueba de Levene para la igualdad de las varianzas difiere según el tipo de idioma en que se ha realizado la prueba. En el caso del español es $<0,05$, lo que supone que las varianzas no son iguales. En cambio, en la lengua extranjera el $p$. valor es $>0,05$, por lo que suponemos que las varianzas son iguales y el valor $p$ calculado -sig. Bilateral- en la prueba de igualdad de medias es menor al nivel de significancia $<0,05$, por lo que se rechaza la hipótesis nula de igualdad de medias. Por tanto, la lectura de prensa está relacionada con la identificación de vocabulario solo en el caso de la lengua extranjera, ya que en lengua materna parece no tener ninguna relación.

Tabla 1. T de Student para la variable "Lectura de prensa" y el léxico disponible

\begin{tabular}{|c|c|c|c|c|c|c|}
\hline & \multicolumn{2}{|c|}{$\begin{array}{l}\text { Prueba de Levene de } \\
\text { calidad de varianzas }\end{array}$} & \multicolumn{3}{|c|}{ Prueba t para la igualdad de medias } \\
\hline & & $\mathbf{F}$ & Sig. & t & gl & $\begin{array}{c}\text { Sig. } \\
\text { (bilateral) }\end{array}$ \\
\hline \multirow{2}{*}{ Español } & $\begin{array}{l}\text { Se asumen varianzas } \\
\text { iguales }\end{array}$ & 4,049 & ,046 & $-1,877$ & 158 & ,062 \\
\hline & $\begin{array}{l}\text { No se asumen va- } \\
\text { rianzas iguales }\end{array}$ & & & $-2,069$ & 99,878 & ,041 \\
\hline \multirow{2}{*}{$\begin{array}{l}\text { Lengua } \\
\text { extranjera }\end{array}$} & $\begin{array}{l}\text { Se asumen varianzas } \\
\text { iguales }\end{array}$ & 1,146 & ,286 & $-3,657$ & 158 & ,000 \\
\hline & $\begin{array}{l}\text { No se asumen va- } \\
\text { rianzas iguales }\end{array}$ & & & $-4,147$ & 31,293 & ,000 \\
\hline
\end{tabular}

\section{DISCUSIÓN Y CONCLUSIONES}

La mayoría de los estudiantes suele dedicar algún tiempo a la semana a leer prensa en español -más del 70\%-siendo mayor el número de horas en el caso de los ELM que los de ELE. Sin embargo, cuando se trata de leer en lengua extranjera, el porcentaje es muy reducido en ambos casos. Gracias a las tecnologías y a los avances del mundo globalizado, muchos periódicos están disponibles en línea de forma gratuita, con lo que el acceso no supondría ningún impedimento y su escaso consumo estaría supeditado a otros factores como la motivación, el interés por hacerlo o incluso el desconocimiento de los diferentes recursos (Parrat, 2010; Spychała-Wawrzyniak, Sagermann Bustinza, 2016).

Este estudio contribuye a reforzar la importancia de la lectura en la adquisición del vocabulario dando un paso más allá y distinguiendo entre los resultados de una prueba sobre léxico activo y pasivo. En el caso del léxico activo, mostrado a través de la prueba, se evidencia una relación no significativa con el consumo de prensa. Estos resultados, si se presta atención a la lengua extranjera, difieren con los de Santos-Díaz 
(2017), en los que la lectura se relacionaba tanto con el léxico pasivo como con el activo. Ello puede deberse a que tan solo un $13,75 \%$ de los estudiantes dedica alguna hora semanal a leer prensa en lengua extranjera.

En cuanto a los resultados de la prueba en español de los estudiantes de ELE, muestran un patrón análogo a los de lengua extranjera de ELM. Si bien no se ha realizado la prueba de comparación de medias debido al escaso número de informantes, este hallazgo abre nuevas vías de investigación en las que se pudiera ampliar la muestra de estos estudiantes, así como de los de naturaleza bilingüe. De esta forma, los datos se podrían segmentar en tres subgrupos: monolingües de español, monolingües de otra lengua y bilingües. Asimismo, se tendría que definir el perfil de la muestra y que resulte interesante para el tipo de programa educativo, material o metodología que se esté evaluando. En nuestro caso, igual que en Herranz (2020), la selección de los participantes atiende a la necesidad de conocer el perfil lingüístico y sociodemográfico del futuro profesorado.

Estos hallazgos son cruciales para diseñar propuestas didácticas en la enseñanza de lenguas. Leer la prensa podría contribuir a eliminar estereotipos marcados por el alumnado que no ha tenido la oportunidad de viajar al contexto en el que se habla el idioma estudiado (Šifrar, 2020). Se recomienda incluir noticias interesantes que motiven a los estudiantes a leer prensa tanto dentro como fuera del aula para maximizar el tiempo de exposición a la lengua extranjera (Fernández, Fernández-Jódar, 2009), acompañadas de metodologías innovadoras (Castillo-Rodríguez, Torrado, 2020). Resulta evidente que, cuanto mayor sea la exposición del alumnado a la lengua extranjera, mayores avances en su competencia lingüística obtendrá y, además, si lo hacen con una buena selección de fuentes, como las que ofrece la prensa, podrán estudiar la lengua desde una visión más pragmática y cultural tanto en el aula como en su tiempo libre (Santos-Díaz, Trigo, Romero, 2020). 


\section{BIBLIOGRAFÍA}

Ávila, A.M., Santos-Díaz, I.C., Trigo, E. (2020). Análisis léxico-cognitivo de la influencia de los medios de comunicación en las percepciones de universitarios españoles ante la COVID-19. Círculo de Lingüistica Aplicada a la Comunicación, 84, 85-95. DOI: 10.5209/clac.70701.

Brown, R., Waring, R., Donkaewbua, S. (2008). Incidental vocabulary acquisition from reading, reading-while-listening, and listening to stories. Reading in a Foreign Language, 20 (2), 136-163.

Castillo-Fadić, M.N., Sologuren, E. (2020). Léxico frecuente, riqueza léxica y estereotipos sobre la lectura de profesores en formación. Logos: Revista de Lingüística, Filosofía y Literatura, 30 (1), 69-85. DOI: $10.15443 /$ RL3006.

Castillo-Rodríguez, C.M., Torrado, M. (2020). Siga o caminho: Uma proposta de aprendizagem para a sala de aula. H2D. Revista De Humanidades Digitais, 2 (1). DOI: 10.21814/h2d.2539.

Cepeda, M., Cárdenas, Á., Carrasco, M., Castillo, N., Flores, J., González, C., Oróstica, M. (2017). Relación entre disponibilidad léxica y comprensión lectora, en un contexto de educación técnico profesional rural. Revista Sophia Austral, 1 (1), 81-93.

Dörnyei, Z., Csizér, K. (2012). How to design and analyze surveys in SLA research? In A. Mackey \& S. Gass (Eds.), Research methods in second language acquisition: A practical guide (pp. 74-94). Malden, MA: Wiley-Blackwell.

Fernández, J., Fernández-Jódar, R. (2009). Uso didáctico de la prensa en las clases de E/LE en contexto universitario. Studia Romanica Posnaniensia, 36, 65-70. DOI: 10.14746/strop.2009.36.005.

Gougenheim, G., Michéa, R., Rivenc, P., Sauvageot, A. (1956). L'élaboration du francais élémentaire. Paris: Didier.

Herranz, C. (2020). Palabra de maestro. Análisis del léxico disponible de los futuros docentes. Berna: Peter Lang.

Jiménez-López, G., Santos-Díaz, I.C., Trigo, E. (2019). La comprensión lectora en lengua extranjera y su relación con las prácticas lectoras. In S. Alonso, J.M. Romero, C. Rodríguez-Jiménez \& J.M. Sola (Eds), Investigación, Innovación docente y TIC. Nuevos Horizontes Educativos (pp. 1724-1738). Madrid: Dikynson.

Juárez, M. (2019). Influencia de la formación inicial del profesorado en los hábitos lectores y en el concepto de educación literaria. Investigaciones Sobre Lectura, 12, 99-115. DOI: 10.37132/isl.v0i12.287.

López-González, A.M. (2014). Disponibilidad léxica. Teoría método y análisis. Lodz: Universidad de Lodz.

Parratt, S. (2010). Consumo de medios de comunicación y actitudes hacia la prensa por parte de los universitarios. Zer: Revista de estudios de comunicación = Komunikazio ikasketen aldizkaria, 15 (28), 133-149.

Romero, M.F., Jiménez, R. (2015). La lectura intensiva como estrategia para el desarrollo de las habilidades comunicativas entre estudiantes de secundaria de ELE. In E. Stala, R.S. Balches \& C. Tatoj (Eds.), Tendencias en la enseñanza de español LE. Perspectivas glotodidácticas y metodológicas contemporáneas (pp. 339-361). Kraków: Wydawnictwo Uniwersytetu Jagiellońskiego.

Romero, M.F., Jiménez, R., Trigo, E. (2020). Donde habita el olvido: recuperando el lugar de la gramática en las aulas. Lublin studies in modern languages and literatura, 44 (3), 117-130. DOI: 10.17951/ 1smll.2020.44.3.117-130.

Samper-Hernández, M. (2002). Disponibilidad léxica en alumnos de español como lengua extranjera. Málaga: ASELE.

Santos-Díaz, I.C. (2017). Incidencia de la lectura en el vocabulario y la ortografía en lengua materna y extranjera. Ocnos, 16, 79-88. DOI: 10.18239/ocnos.

Santos Díaz, I.C. (2020). El léxico bilingüe del futuro profesorado. Análisis y pautas para estudios de disponibilidad. Berlín: Peter Lang. 
Santos-Díaz, I.C., Trigo, E., Romero, M.F. (2020). Relación entre la exposición informal a una lengua extranjera (inglés y francés) y la competencia léxica. In I. Karapetjana (Ed.), Language for International Communication: Linking Interdisciplinary Perspectives, vol. 3 (pp. 431-439). Riga: University of Latvia.

Spychała-Wawrzyniak, M. (2019). El papel de las emociones en la enseñanza intercultural del español como lengua extranjera (ELE). Studia Romanica Posnaniensia, 46 (4), 163-172. DOI: 10.14746/ strop.2019.464.014.

Spychała-Wawrzyniak, M., Sagermann Bustinza, L. (2016). La motivación de los alumnos polacos para el aprendizaje del español como lengua extranjera. Studia Romanica Posnaniensia, 43 (3), 85-102. DOI: 10.14746/strop.2016.433.006.

Šifrar, M. (2014). Disponibilidad léxica en distintos niveles de español/Lengua extranjera. Studia Romanica Posnaniensia, 41 (1), 63-85. DOI: 10.14746/strop.2014.411.005.

Šifrar, M. (2020). Estereotipos culturales sobre España en estudiantes universitarios extranjeros: el caso de los Erasmus eslovenos. Ogigia. Revista electrónica de estudios hispánicos, 27, 213-234. DOI: 10.24197/ogigia.27.2020.213-234.

Trigo, E., Romero, M.F. (2017). Geografía literaria en el aula de ELE: una aproximación al Cádiz de 1812 desde la ruta literaria de las libertades. Studia Iberystyczne, 16, 283-301. DOI: 10.12797/ SI.16.2017.16.17.

Villegas-Paredes, G. (2020). El articuento y el tratamiento de contenidos socioculturales en ELE: propuesta metodológica desde el enfoque intercultural. Tejuelo, 32, 299-330. DOI: 10.17398/19888430.32.299. 